\title{
Uniportal versus multiport video-assisted thoracoscopic surgery for anatomical lung resections: a glance at a dilemma
}

\author{
Ezel Erşen ${ }^{1}$, Burcu Kılıç ${ }^{1}$ Hasan Volkan Kara ${ }^{1}$, Mehlika İşcan $^{1}$, Nurlan Alizade ${ }^{1}$, Ahmet Demirkaya ${ }^{2}$, Akif Turna ${ }^{1}$, \\ Kamil Kaynak ${ }^{1}$ \\ ${ }^{1}$ Department of Thoracic Surgery, Cerrahpaşa Medical Faculty, Istanbul University, Istanbul, Turkey \\ ${ }^{2}$ Department of Thoracic Surgery, School of Medicine, Istanbul Acıbadem University, Istanbul, Turkey
}

Videosurgery Miniinv 2018; 13 (2): 215-220

DOI: https://doi.org/10.5114/wiitm.2018.75897

\begin{abstract}
Introduction: As the number of operations performed by videothoracoscopy is increasing, there is also a tendency to decrease the number of port incisions. Apart from the reduced number of surgical incisions, there are a few reports and systematic reviews that demonstrate some potential advantages of the uniportal video-assisted thoracoscopic surgery, but the impact of the reduced incisions in the clinical setting still remains uncertain.

Aim: To compare uniportal video-assisted thoracoscopic surgery to multiport video-assisted thoracoscopic surgery for anatomical lung resections in patients with malignant and benign lung diseases.

Material and methods: From August 2010 to April 2016, a total of 102 patients with malignant and benign lung diseases underwent videothoracoscopic lobar and sublobar lung resections in our department. Comorbidities, tumor stage, tumor localization, mortality, operative time, pain visual analogue scale, length of hospital stay, perioperative blood loss, duration and amount of postoperative drainage and air leak, number of harvested lymph nodes and complication rates were analyzed.

Results: No significant difference was found in the duration of chest tube drainage, pain visual analogue scale score, length of hospital stay, perioperative blood loss, amount of postoperative drainage, number of harvested lymph nodes or complication rate. There was no surgical mortality in either of the two groups. However, operative time was shorter (189 min vs. $256 \mathrm{~min}, p<0.005$ ) in the multiport group than in the uniportal group.

Conclusions: Compared with the uniportal approach, the multiport approach is associated with a significantly shorter operative time in our study.
\end{abstract}

Key words: uniportal, multiport, video-assisted thoracoscopic surgery.

\section{Introduction}

Minimally invasive techniques in thoracic surgery are feasible, while they are also associated with less postoperative morbidity and fast recovery. As the number of operations performed by video-assisted thoracoscopic surgery (VATS) is increasing, there is also a tendency to decrease the number of port incisions. Thoracic surgeons used to perform resections by conventional open thoracotomy until the evolution of VATS in the early 1990s [1, 2].

In time, growing evidence suggested that VATS lobectomy with systematic lymph node dissection was a good alternative in patients with early stage lung cancer. On the other hand, VATS had advantages such as less deterioration in pulmonary function, less postoperative pain, shorter hospital stay, lower morbidity and nearly equal long-term oncological

\section{Address for correspondence}

Ezel Erşen, Department of Thoracic Surgery, Cerrahpaşa Medical Faculty, Istanbul University, 34098 Istanbul, Turkey,

phone: +90 5057311567, e-mail: drezelersen@gmail.com 
outcomes in comparison with conventional thoracotomy [3].

In addition, VATS sublobar resections have been performed in recent years on highly selected patients with early stage lung cancer, who had comorbidities or impaired pulmonary functions. It has also been regarded as an alternative to nonsurgical therapy with a comparable local relapse rate and nonsignificant difference of 5-year survival in uncontrolled trials [4-7].

Video-assisted thoracic surgery has evolved rapidly in the last two decades. Together with the advances in thoracoscopic techniques, the number of incisions used during VATS has decreased from three or four to one. Rocco et al. published the first paper about the use of uniportal VATS for pulmonary

Table I. Demographic and clinical characteristics of patients before surgery

\begin{tabular}{|c|c|}
\hline Parameter & Value \\
\hline Age, median (range) [years] & $61.3(19-80)$ \\
\hline \multicolumn{2}{|l|}{ Gender, $n(\%)$ : } \\
\hline Male & $69(68)$ \\
\hline Female & $33(32)$ \\
\hline \multicolumn{2}{|l|}{ Comorbidity, $n(\%)$ : } \\
\hline Diabetes mellitus & $13(15)$ \\
\hline Hypertension & $28(32)$ \\
\hline Coronary heart disease & $20(23)$ \\
\hline COPD & $10(11)$ \\
\hline Renal dysfunction & $7(8)$ \\
\hline Arrhythmia & $5(6)$ \\
\hline Asthma & $4(4)$ \\
\hline \multicolumn{2}{|l|}{ Histological type, $n$ : } \\
\hline Adenocarcinoma & 47 \\
\hline Squamous cell carcinoma & 20 \\
\hline Adenosquamous cell & 3 \\
\hline Large cell & 3 \\
\hline Carcinoid cancer & 8 \\
\hline Bronchiectasis & 11 \\
\hline Others & 9 \\
\hline $\mathrm{FEV}_{1}$, mean $(\%)$ & $2350 \mathrm{ml}(84.7)$ \\
\hline
\end{tabular}

$F E V_{1}$-forced expiratory volume in $1 \mathrm{~s}$. wedge resection in 2004 [8], and in 2011 Gonzalez et al. reported a case of left lower lobectomy performed by a video-assisted single-port incision [9]. Since then, the uniportal VATS technique has become an even less invasive alternative to the conventional multiport approach.

Apart from the reduced number of surgical incisions, there are institutional reports and recent systematic reviews that demonstrate some potential advantages of the uniportal VATS such as reduced postoperative pain and paresthesia and more post-operative satisfaction, although the impact of the reduced incisions in the clinical setting remains uncertain $[10,11]$.

In this study, we aimed to compare uniportal VATS to multiport VATS for anatomical lung resections in patients with malignant and benign lung diseases. Endpoints included comorbidities, tumor stage, tumor localization, mortality, operative time, pain visual analogue scale (VAS), length of hospital stay, perioperative blood loss, duration and amount of postoperative drainage and air leak, number of harvested lymph nodes as well as complication rates.

\section{Aim}

The aim of this study was to compare the outcomes of uniportal VATS to multiport VATS for anatomical lung resections in patients with malignant and benign lung diseases in a clinical setting.

\section{Material and methods}

From August 2010 to April 2016, a total of 102 patients with malignant and benign lung diseases (69 male (68\%), 33 female (32\%)) underwent videothoracoscopic lobar and sublobar lung resections in our department. The mean age was 61.3 years (range: 19-80 years). The demographic data of the patients are presented in Table I. Electrocardiogram, pulmonary function tests, chest computed tomography (CT) scan and bronchoscopy were performed in all patients, while a brain magnetic resonance imaging (MRI) was performed in patients with malignant disease. Positron emission tomography-CT (PET-CT) was also performed when routine tests could not rule out an underlying metastasis. Comorbidities, tumor stage, tumor localization, mortality, operative time, pain VAS postoperative day 1 , length of hospital stay, perioperative blood loss, duration and amount of postoperative drainage and air leak, number of 
harvested lymph nodes as well as complication rates and rates of conversion to open thoracotomy were collected for the database.

Intra-operative and post-operative pain management was the same in both groups. In addition, the same intravenous analgesic protocol was administered to both groups. A VAS score of ' 0 ' indicated no pain, while a score of ' 10 ' indicated severe pain. The chest drain was withdrawn in cases where the 24-hour drainage volume was less than $100 \mathrm{ml}$ and postoperative chest X-ray showed no abnormality. The discharge criteria were as follows: (I) patients with normal clinical status and recovered mobility status; (II) no obvious fever after chest tube withdrawal.

All patients underwent general anesthesia, while each patient was intubated with a double-lumen endotracheal tube to accomplish single lung ventilation. The patients' vital signs were followed and noted throughout the operation.

\section{Operative procedure}

The patient was placed in the lateral decubitus position, while the operator stood at the anterior side of the patient. For triportal VATS, one $1.5 \mathrm{~cm}$ port incision for viewing was made at the seventh intercostal space on the middle axillary line. Two additional ports were opened for working: one $4 \mathrm{~cm}$ operation port on the fourth intercostal space of the anterior axillary line and one $2 \mathrm{~cm}$ port on the posterior axillary line. As a modification, we used the posterior port a little anteriorly in order to reduce the postoperative pain.

For biportal (hybrid) VATS, one $5 \mathrm{~cm}$ incision was made on the fourth or fifth intercostal space along the anterior axillary line and one $1.5-2 \mathrm{~cm}$ incision was made for viewing on the seventh intercostal space on the midaxillary line. For the single-port group, one $3.5-4.5 \mathrm{~cm}$ incision was made on the fourth or fifth intercostal space along the anterior axillary line. A plastic wound protector was used for the operation port incision to avoid surgical site infection and tumor spread. Standard lobectomy and segmentectomy were performed, while additional hilar and mediastinal lymph node dissection was also achieved. Endoscopic bipolar vessel-sealing devices or hemoclips were used for small vessels, while an endoscopic stapler was used to deal with the great vessels and the bronchus. We usually re- sected the bronchus at the final stage of lobectomy or sublobectomy. After resection, the specimen was placed in an endoscopic plastic bag under thoracoscopic assistance and later removed through the operational incision.

\section{Statistical analysis}

Statistical analysis was performed using Pearson's $\chi^{2}$ test for bivariate analysis. All statistical analyses were performed using IBM SPSS Statistics, version 20.0 (IBM Corp., Armonk, N.Y.). Values of $p<0.05$ were considered statistically significant.

\section{Results}

Between August 2010 and April 2016, a total of 102 patients underwent anatomical VATS pulmonary resection. Triportal technique was used in 47 (46\%) patients, while biportal technique was used in 34 (33\%) patients and uniportal technique was used in 21 (20\%) patients. In the uniportal group, an additional port was required in 5 patients (bleeding in 2 patients and severe adhesions in 3 patients). The conversion rate to open thoracotomy in the series was found to be $3.9 \%-4$ patients (3 bleeding, 1 severe adhesions). Ninety-one lobectomy, 2 pneumonectomy and 9 segmentectomy operations were performed. Out of the 102 patients who underwent VATS pulmonary resection, 73 of them had non-small cell lung carcinoma (NSCLC), while 8 patients had carcinoid tumors, 11 patients had bronchiectasis and 9 patients had other lesions. The demographics, clinical characteristics, perioperative-postoperative characteristics, operative details, and tumor characteristics of the 102 patients are described in Tables I-III.

No significant difference was found in the duration of chest tube drainage, pain VAS score, length of hospital stay, perioperative blood loss, amount of postoperative drainage, number of harvested lymph nodes or complication rate. There was no surgical mortality in either of the two groups. However, operative time was shorter (189 min vs. $256 \min , p<0.005$ ) in the multiport group than in the uniportal group.

\section{Discussion}

The uniportal thoracoscopic technique was first described by Migliore in patients with pleural diseases in 2003 [12]. In 2004 Rocco et al. report- 
Table II. Comparison of perioperative and postoperative parameters between uniportal and multiport group

\begin{tabular}{|lccc|}
\hline Parameter & Uniportal & Multiport & $P$-value \\
\hline Operation time* [min] & 256 & 189 & 0.0003 \\
\hline Perioperative blood loss [ml] & 300 & 224 & 0.883 \\
\hline Chest tube duration [day] & 5.6 & 4.5 & 0.180 \\
\hline Postoperative drainage [ml] & 697.5 & 683 & 0.939 \\
\hline Hospital stay [day] & 6.9 & 5.9 & 0.392 \\
\hline Post-op VAS score & 3.1 & 2.8 & 0.620 \\
\hline Harvested (N1) lymph nodes & 10.3 & 8.7 & 0.284 \\
\hline Harvested (N2) lymph nodes & 6.2 & 8.04 & 0.401 \\
\hline Postoperative complications: & 6 & 8 & 0.554 \\
\hline Air leakage & 4 & 5 & 2 \\
\hline Pneumonia & 1 & 1 & \\
\hline Arrhythmia & 1 & & \\
\hline${ }^{*}$ Operation time ranged from incision to suturing the skin, including the time for frozen section. & \\
\hline
\end{tabular}

Table III. Tumor characteristics

\begin{tabular}{|c|c|}
\hline Parameter & Value \\
\hline Tumor size, mean (range) [cm] & $2.9(0.1-10)$ \\
\hline \multicolumn{2}{|l|}{ Tumor location, $n$ : } \\
\hline LUL & 19 \\
\hline LLL & 24 \\
\hline RUL & 26 \\
\hline RML & 10 \\
\hline RLL & 17 \\
\hline \multicolumn{2}{|l|}{ Operative procedure, $n(\%)$ : } \\
\hline Lobectomy & $92(90.1)$ \\
\hline Segmentectomy & $9(8.8)$ \\
\hline \multicolumn{2}{|l|}{ Staging, $n(\%)$ : } \\
\hline $\mathrm{A}$ & $28(34.1)$ \\
\hline IB & $27(32.9)$ \\
\hline$\| A$ & $13(15.8)$ \\
\hline$\| B$ & $6(7.3)$ \\
\hline$\| \mathrm{A}$ & $6(7.3)$ \\
\hline IV (solitary met.) & $2(2.4)$ \\
\hline \multicolumn{2}{|l|}{ T stage, $n(\%)$ : } \\
\hline $\mathrm{T} 1 \mathrm{a}$ & $25(31)$ \\
\hline $\mathrm{T} 1 \mathrm{~b}$ & $7(8)$ \\
\hline $\mathrm{T} 2 \mathrm{a}$ & $38(47)$ \\
\hline $\mathrm{T} 2 \mathrm{~b}$ & $3(4)$ \\
\hline T3 & $8(10)$ \\
\hline
\end{tabular}

ed pulmonary wedge resection with the uniportal approach [8], while a few years later Gonzales et al. published their experience in uniportal VATS lobectomy and lymphadenectomy [13]. Since then, the uniportal approach has been performed routinely in many other major thoracic interventions [14-17].

Most available literature has reported the feasibility and safety of uniportal thoracoscopic lobectomy, although there is still much debate about which approach is the appropriate choice for the patients. There are few studies in the literature that compare these two techniques. On the other hand, until now there has only been one randomized controlled study comparing the effectiveness and prognosis between the uniportal VATS and multiport VATS [18-28]. There are also a few systematic reviews and metaanalyses that focus on this subject $[10,11]$.

In our study, we found that the outcomes of uniportal VATS for anatomical lung resections were comparable to those in the multiport VATS group.

In a recent meta-analysis, eleven studies were identified in the databases of PubMed, Cochrane Library, SpringerLink and ScienceDirect. Randomized controlled trials and non-randomized studies evaluating the outcomes of uniportal versus multiport VATS in the treatment of lung cancer were analyzed [10]. A total of 1314 patients (649 in the uniportal group and 665 in the multiport group) were included. In this study, there was no difference between the two groups in terms of the operative time. The 
amount of blood loss and duration of chest tube drainage were also similar. Postoperative hospital stay was found to be shorter in the uniportal group $(p<0.008)$. These results suggested that the number of harvested lymph nodes were similar in the two approaches, while the overall complication rate was lower in the uniportal group. However, no significant association was found for common complications such as prolonged air leak, pneumonia, atelectasis or arrhythmia. In our study, the number of harvested lymph nodes was similar in both groups: number of N1 nodes - uniportal 10.3 vs. multiport 8.7 $(p=0.284)$; number of N2 nodes - uniportal 6.2 vs. multiport $8.04(p=0.401)$. In a recent study, $\mathrm{Mu}$ et al. reported a conversion rate of $3.4 \%$ for the uniportal approach and $2.3 \%$ for the triportal approach [25]. The overall conversion rate in our study was $3.9 \%(6.3 \%$ for the multiport VATS group and $4.7 \%$ for the uniportal VATS group, $p=0.547$ ). Six studies in the meta-analysis explored postoperative pain. While only three of them found that the uniportal approach was associated with less postoperative pain, the other studies did not indicate any significant association. We did not find any difference in postoperative pain between these two groups, either (mean VAS score: uniportal 3.1 vs. multiport 2.8, $p=0.620$ ). Although our study has similarities with the meta-analysis, we found that multiport anatomical pulmonary resection might be associated with shorter operative time. However, the meaning of these data might be controversial, since a surgical procedure requires a sufficient learning curve for experienced thoracic surgeons to perform the major anatomical lung resections.

The only randomized study, by Perna et al., concluded that uniportal VATS lobectomy does not provide better postoperative outcomes than other VATS techniques [28].

Similarly, we did not find any major difference between the two groups in the clinical setting. Our results are compatible with the literature considering the outcomes of uniportal and multiport VATS groups. On the other hand, there are several limitations in our study as well. For instance, the number of patients who underwent uniportal VATS pulmonary resection was relatively small. In addition, we analyzed the data from one medical center, which restricts the generalization of our conclusions. Lastly, our study did not include the analysis of long-term survival outcomes. Hence, our results need further validation in a randomized controlled clinical trial with a larger number of patients.

\section{Conclusions}

Uniportal VATS is at least as safe and effective as multiport VATS for anatomical resections in patients with malignant and benign lung disease. Outcomes of uniportal VATS anatomical lung resection are comparable to the multiport approach in our single medical center series. In our series, the only statistically significant difference found between the two techniques was for duration of the operation. Compared with the uniportal approach, the multiport approach is associated with a significantly shorter operative time. On the other hand, the question as to whether one of these techniques is superior to the other still remains unclear. Likewise, prospective randomized controlled studies are required in order to compare the uniportal VATS with multiport VATS in terms of intermediate- and long-term results.

\section{Conflict of interest}

The authors declare no conflict of interest.

\section{References}

1. McKenna RJ Jr, Houck W, Fuller CB. Video-assisted thoracic surgery lobectomy: experience with 1,100 cases. Ann Thorac Surg 2006; 81: 421-6.

2. Swanson SJ, Herndon JE 2nd, D’Amico TA, et al. Video-assisted thoracic surgery lobectomy: report of CALGB 39802: a prospective, multi-institution feasibility study. J Clin Oncol 2007; 25: 4993-7.

3. Yan TD, Black D, Bannon PG, et al. Systematic review and meta-analysis of randomized and nonrandomized trials on safety and efficacy of video-assisted thoracic surgery lobectomy for early-stage nonsmall-cell lung cancer. J Clin Oncol 2009; 27: 2553-62.

4. Howington JA, Blum MG, Chang AC, et al. Treatment of stage I and || non-small cell lung cancer: diagnosis and management of lung cancer: American College of Chest Physicians evidence-based clinical practice guidelines. Chest 2013; 143 : 278-313.

5. Cao C, Chandrakumar D, Gupta S, et al. Could less be more? A systematic review and meta-analysis of sublobar resections versus lobectomy for non-small cell lung cancer according to patient selection. Lung Cancer 2015; 89: 121-32.

6. Zhong C, Fang W, Mao T, et al. Comparison of thoracoscopic segmentectomy and thoracoscopic lobectomy for small-sized stage IA lung cancer. Ann Thorac Surg 2012; 94: 362-7.

7. Carr SR, Schuchert MJ, Pennathur A, et al. Impact of tumor size on outcomes after anatomic lung resection for stage $1 \mathrm{~A}$ non- 
small cell lung cancer based on the current staging system. J Thorac Cardiovasc Surg 2012; 143: 390-7.

8. Rocco G, Martin-Ucar A, Passera E. Uniportal VATS wedge pulmonary resections. Ann Thorac Surg 2004; 77: 726-8.

9. Gonzalez D, Delgado M, Paradela M, et al. Uniincisional videoassisted thoracoscopic left lower lobectomy in a patient with an incomplete fissure. Innovations 2011; 6: 45-7.

10. Harris CG, James RS, Tian DH, et al. Systematic review and meta-analysis of uniportal versus multiportal video-assisted thoracoscopic lobectomy for lung cancer. Ann Cardiothorac Surg 2016; 5: 76-84.

11. Yang Z, Shen Z, Zhou Q, et al. Single-incision versus multiport video-assisted thoracoscopic surgery in the treatment of lung cancer: a systematic review and meta-analysis. Acta Chir Belg 2017; 118: 85-93.

12. Migliore M. Efficacy and safety of single-trocar technique for minimally invasive surgery of the chest in the treatment of noncomplex pleural disease. J Thorac Cardiovasc Surg 2003; 126: 1618-23.

13. Gonzalez D, de la Torre M, Paradela M, et al. Video-assisted thoracic surgery lobectomy: 3-year initial experience with 200 cases. Eur J Cardiothorac Surg 2011; 40: 21-8.

14. Gonzalez-Rivas D, Fieira E, Mendez L, et al. Single-port video-assisted thoracoscopic anatomic segmentectomy and right upper lobectomy. Eur J Cardiothorac Surg 2012; 42: 169-71.

15. Gonzalez-Rivas D, de la Torre M, Fernandez R, et al. Single-incision video-assisted thoracoscopic right pneumonectomy. Surg Endosc 2012; 26: 2078-9.

16. Gonzalez-Rivas D, Fieira E, de la Torre M, et al. Bronchovascular right upper lobe reconstruction by uniportal video-assisted thoracoscopic surgery. I Thorac Dis 2014; 6: 861-3.

17. Gonzalez-Rivas D, Delgado M, Fieira E, et al. Single-port video-assisted thoracoscopic lobectomy with pulmonary artery reconstruction. Interact Cardiovasc Thorac Surg 2013; 17: 889-91.

18. Li C, Ma H, He J, et al. Clinical analysis of thoracoscopic lobectomy in the treatment of peripheral lung cancer with single utility port. Chin J Lung Cancer 2013; 16: 487-91.

19. Chung JH, Choi YS, Cho JH, et al. Uniportal video-assisted thoracoscopic lobectomy: an alternative to conventional thoracoscopic lobectomy in lung cancer surgery? Interact Cardiovasc Thorac Surg 2015; 20: 813-9.

20. Wang BY, Liu CY, Hsu PK, et al. Single-incision versus multiple-incision thoracoscopic lobectomy and segmentectomy: a propensity-matched analysis. Ann Surg 2015; 261: 793-9.

21. Zhu Y, Liang $M, W u W$, et al. Preliminary results of single-port versus triple-port complete thoracoscopic lobectomy for nonsmall cell lung cancer. Ann Transl Med 2015; 3: 92.

22. Liu CC, Shih CS, Pennarun N, et al. Transition from a multiport technique to a single-port technique for lung cancer surgery: is lymph node dissection inferior using the single-port technique? Eur J Cardiothorac Surg 2016; 49 Suppl 1: 64-72.

23. Hirai K, Takeuchi S, Usuda J. Single-incision thoracoscopic surgery and conventional video-assisted thoracoscopic surgery: a retrospective comparative study of perioperative clinical outcomes. Eur J Cardiothorac Surg 2016; 49 Suppl 1: 37-41.

24. Shen $\mathrm{Y}$, Wang $H$, Feng $M$, et al. Single- versus multiple-port thoracoscopic lobectomy for lung cancer: a propensity-matched study. Eur J Cardiothorac Surg 2016; 49 Suppl 1: 48-53.
25. Mu JW, Gao SG, Xue Q, et al. A matched comparison study of uniportal versus triportal thoracoscopic lobectomy and sublobectomy for early-stage non-small cell lung cancer. Chin Med J 2015; 128: 2731-5.

26. McElnay PJ, Molyneux M, Krishnadas R, et al. Pain and recovery are comparable after either uniportal or multiport video-assisted thoracoscopic lobectomy: an observation study. Eur J Cardiothorac Surg 2015; 47: 912-5.

27. French DG, Thompson C, Gilbert S, et al. Transition from multiple port to single port video-assisted thoracoscopic anatomic pulmonary resection: early experience and comparison of perioperative outcomes. Ann Cardiothorac Surg 2016; 5: 92-9.

28. Perna V, Carvajal AF, Torrecilla JA, et al. Uniportal video-assisted thoracoscopic lobectomy versus other video-assisted thoracoscopic lobectomy techniques: a randomized study. Eur J Cardiothorac Surg 2016; 50: 411-5.

Received: 9.01.2018, accepted: 4.03.2018. 